\title{
Optically tailored access to metastable electronic states
}

\author{
David L. Andrews* , David S. Bradshaw \\ School of Chemistry, University of East Anglia, Norwich Research Park, Norwich NR4 7TJ, United Kingdom
}

\section{A R T I C L E I N F O}

\section{Article history:}

Received 21 September 2013

In final form 26 October 2013

Available online 4 November 2013

\begin{abstract}
A B S T R A C T
On irradiating a molecular system with a laser beam of ultraviolet or visible frequency, photon absorption occurs when an electronic state is at a suitable energy level relative to an initial state. Despite meeting this criterion, interesting metastable states can remain inaccessible because of symmetry constraints. In this Letter a mechanism, based on the input of an off-resonant beam, is shown to enable the population of such states. This is achievable because the laser-modified process involves different selection rules compared to conventional photon absorption. The effects of applying the stimulus beam to either a one- or two-photon process are examined.
\end{abstract}

(c) 2013 Elsevier B.V. All rights reserved.

\section{Introduction}

In conventional single-photon molecular spectroscopy, absorption arising from the application of a resonant beam involves individual molecule-photon interactions generally defined by first order time-dependent perturbation theory $[1,2]$. Under normal circumstances, higher order contributions are essentially insignificant since they simply denote self-energy corrections. However, this is no longer the case when the system is irradiated by an off-resonant laser beam of sufficient intensity - specifically a stimulus beam with an optical frequency at which the molecule is transparent. Then it becomes possible to engage, as part of the resonant absorption, elastic forward-scattering of the off-resonant stimulus photons. No net absorption or stimulated emission of the stimulus beam occurs; these photons are annihilated and created back into the same radiation state so that the beam emerges unchanged (Figure 1). This mechanism, entailing three molecule-photon interactions, relates to a significant higher order contribution, the result of which is that the transition dipole moment corresponding to single-photon absorption is modified.

Similar considerations can apply to the case of two-photon absorption. The absorption of two identical photons by a molecule, via interaction with a resonant laser beam, is primarily considered a second order contribution. However, irradiating this system with a stimulus beam, of sufficient intensity, again affects the resonant multiphoton absorption of light. This fourth-order process entails four molecule-photon interactions (Figure 2) and is analogous to the one-photon case.

This Letter details a substantial effect resulting from these higher order processes. Specifically, it is shown how the off-resonant beam empowers electronic transitions that are normally forbidden.

\footnotetext{
* Corresponding author. Fax: +44 1603592003.

E-mail addresses: d.l.andrews@uea.ac.uk, david.andrews@physics.org (D.L. Andrews).
}

Although the energy of either one or two photons within the excitation beam may match the difference between the energy levels of two electronic states (the lower of which is populated), absorption will usually not occur due to symmetry considerations. These are states that, nevertheless, may be populated due to higher order processes since different selection rules apply for any such case. The following analysis investigates electronic states that are inaccessible to one- and two-photon absorption, but which become available upon throughput of the off-resonant beam. Such a situation may indeed apply, in certain cases, to lowest energy electronic states: special interest then attaches to the fact that fluorescence emission will normally be forbidden, once the stimulus beam has left the system. Providing non-radiative relaxation is relatively weak, sample molecules may thus remain in states that are unusually long-lived.

\section{Laser-modified one-photon absorption}

\subsection{Expression for efficiency}

The efficiency of laser-modified one-photon absorption, $\Gamma_{1 \varphi}$, is determined from the Fermi Rule [3] so that $\Gamma_{1 \phi} \propto\left|M_{F I}^{(1)}+M_{F I}^{(3)}\right|^{2}$, where $M_{F I}^{(1)}$ and $M_{F I}^{(3)}$ are quantum amplitudes for the single-photon (first-order) and stimulus beam applied (third-order) interaction processes, respectively. Following from the quadratic dependence on these quantum amplitudes, this equation not only includes terms corresponding to first- and third-order perturbations, but also a cross-term relating to quantum interference.

The explicit form of $\Gamma_{1 \varphi}$ has recently been derived elsewhere [4], and is given by:

$$
\begin{aligned}
\Gamma_{1 \phi}= & \left(\frac{\pi \rho I}{c \hbar \varepsilon_{0}}\right) e_{i} \bar{e}_{j}\left[\mu_{i}^{(0) \alpha 0} \bar{\mu}_{j}^{(0) \alpha 0}+\left(I^{\prime} / c \varepsilon_{0}\right) \mu_{i}^{(0) \alpha 0} \bar{\mu}_{j}^{(2) \alpha 0}\left(\omega^{\prime}\right)\right. \\
& \left.+\left(I^{\prime 2} / 4 c^{2} \varepsilon_{0}^{2}\right) \mu_{i}^{(2) \alpha 0}\left(\omega^{\prime}\right) \bar{\mu}_{j}^{(2) \alpha 0}\left(\omega^{\prime}\right)\right],
\end{aligned}
$$




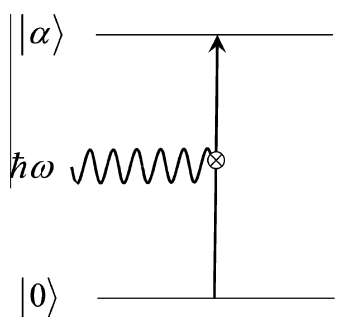

(a)

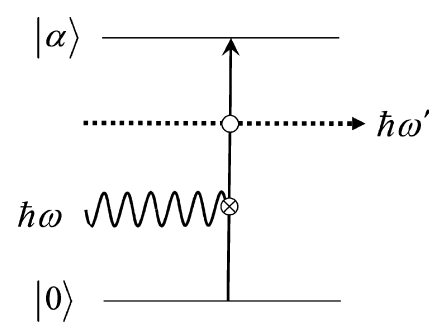

(b)
Figure 1. Energy level representation for: (a) one-photon absorption, the linear interaction, (b) the nonlinear interactions of laser-modified one-photon absorption. Molecular states are signified by horizontal lines with labels $|0\rangle$ and $|\alpha\rangle$ designating ground and excited states, respectively, the wavy line denotes an incoming resonant photon $(\hbar \omega)$ and the vertical arrow is a transition due to the resulting light absorption. The nonlinear case also involves the stimulus laser beam $\left(\hbar \omega^{\prime}\right)$ shown by the horizontal dashed arrow; the crossed and open dot symbolizes one and two molecule-photon interaction, respectively.

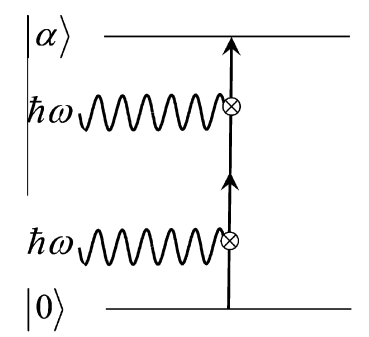

(a)

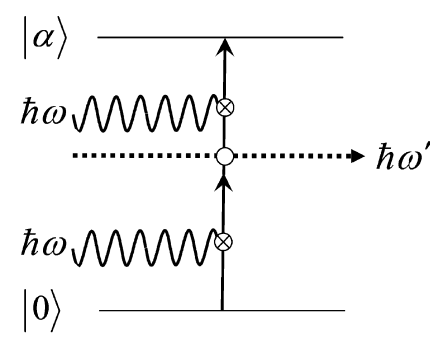

(b)
Figure 2. Energy level representation for: (a) two-photon absorption, (b) the modified nonlinear interactions of two-photon absorption.

where $I$ and $I^{\prime}$ are the intensities of the absorbing (resonant) beam and the throughput laser irradiance, respectively, and $\mathbf{e}$ is the polarization of the resonant beam - the overbar allowing for the possibility of circular polarization. The first term of Eq. (1) relates to one-photon absorption associated with an effective excitation dipole whose zeroth order contribution (independent of the stimulus beam) is designated $\boldsymbol{\mu}^{(0) \alpha 0} \equiv \boldsymbol{\mu}^{\alpha 0}$, where the superscript denotes a $\alpha \leftarrow 0$ transition. The third term corresponds to a contribution quadratic in the electric field of the stimulus beam as given by $\boldsymbol{\mu}^{(2) \alpha 0}\left(\omega^{\prime}\right) \equiv \mathbf{e}^{\prime} \overline{\mathbf{e}}^{\prime} \boldsymbol{\beta}^{\alpha 0}\left(\omega^{\prime}\right)$, where $\mathbf{e}^{\prime}$ is the polarization of the stimulus beam and $\boldsymbol{\beta}^{\alpha 0}\left(\omega^{\prime}\right)$ is a dynamic hyperpolarizability tensor. The former represents direct resonant absorption, and the latter a nonlinear correction term that couples with the off-resonant radiation of frequency $\omega^{\prime}$ (corresponding to elastic forward-scattering). The stimulus beam thus effects a modification of the excitation dipole moment through $\boldsymbol{\mu}^{(2) \alpha 0}\left(\omega^{\prime}\right)$. Finally, it is the second term (linear in $I^{\prime}$ ), signifying a quantum interference of these two amplitudes, that represents the leading correction.

\subsection{Population of 'one-photon forbidden' states}

Certain single-photon absorption processes, symmetry forbidden by electric dipole selection rules, may become allowed if the necessary symmetry is present. Of these electronic transitions, some would be allowed by the selection rules for two-photon absorption, from a beam of half the optical frequency, but others would not be allowed by either single-photon or two-photon processes. Since one-photon processes are also three-photon allowed (although the converse is not necessarily the case - which is of crucial importance for laser-modified one-photon absorption) the normally unattainable states may be activated on input of the off-resonant beam.
The first step to identify electronic transitions that are singlephoton forbidden by symmetry, but become photo-activated on application of the stimulus beam, is to determine the connectivity of the relevant of the radiation and material tensors in Eq. (1). In particular, the key symmetry criteria can be elicited by developing the irreducible tensor formalism for the corresponding tensor components [5-10]. We adopt Cartesian irreducible tensor forms since (compared to the use of spherical tensors) these are very much more readily interpreted and related to molecular geometry, as will emerge. Of particular interest are $\boldsymbol{\mu}^{(0) \alpha 0}$, representing Cartesian components of the electric dipole transition moment due to resonant absorption - formally a rank-1 polar tensor - and $\boldsymbol{\mu}^{(2) \alpha 0}\left(\omega^{\prime}\right)$, components of another rank-1 polar tensor representing the influence of the stimulus radiation. The latter is now recast as:

$\boldsymbol{\mu}^{(2) \alpha 0}\left(\omega^{\prime}\right) \equiv\left[\boldsymbol{\beta}^{\alpha 0}\left(\omega^{\prime}\right): \mathbf{S}\right]_{i}$,

where $\mathbf{S} \equiv e_{j}^{\prime} \bar{e}_{k}^{\prime}$ is an even parity rank-2 tensor signifying the secondorder electric field influence of the stimulus beam, and $\boldsymbol{\beta}^{\alpha 0}\left(\omega^{\prime}\right)$ is a rank-3 material response tensor. The colon in Eq. (2) signifies tensor inner contraction, effected by summation over paired Cartesian indices. Generally, the material response tensor lacks index symmetry and, as a consequence, it may be decomposed into irreducible parts of weights $0^{-}, 1^{-}, 2^{-}$and $3^{-}$, all of odd parity, i.e.:

$\boldsymbol{\beta}^{\alpha .0}=\left[\boldsymbol{\beta}^{\alpha 0}\right]^{(0-)}+\left[\boldsymbol{\beta}^{\alpha 0}\right]^{(1-)}+\left[\boldsymbol{\beta}^{\alpha 0}\right]^{(2-)}+\left[\boldsymbol{\beta}^{\alpha 0}\right]^{(3-)}$,

with frequency dependence now suppressed for clarity. In sequence, from left to right, the four terms of Eq. (3) (all embedded in rank-3 tensor space) are defined as follows: a pseudoscalar; a polar vector; an odd-parity, symmetric and traceless second-rank tensor; a polar traceless and fully index-symmetric third rank tensor. The detailed component forms for each of these contributions are given elsewhere [11].

A similar reduction of the stimulus tensor into components yields:

$\mathbf{S}=[\mathbf{S}]^{(0+)}+[\mathbf{S}]^{(1+)}+[\mathbf{S}]^{(2+)}$,

where successive terms on the right transform as: a scalar; an axial vector; an even-parity traceless and symmetric second-rank tensor. In this instance, it is worth presenting the explicit form of the corresponding components under two separate conditions: for a linearly polarized stimulus beam:

$[\mathbf{S}]_{i j}^{(0+)}=\frac{1}{3} \delta_{i j},[\mathbf{S}]_{i j}^{(1+)}=0,[\mathbf{S}]_{i j}^{(2+)}=e_{i}^{\prime} e_{j}^{\prime}-\frac{1}{3} \delta_{i j}$,

and a circularly polarized off-resonant laser:

$[\mathbf{S}]_{i j}^{(0+)}=\frac{1}{3} \delta_{i j},[\mathbf{S}]_{i j}^{(1+)}=\frac{i}{2} \varepsilon_{i j k} \hat{k}_{k}^{\prime},[\mathbf{S}]_{i j}^{(2+)}=\frac{1}{6} \delta_{i j}-\frac{1}{2} \hat{k}_{i}^{\prime} \hat{k}_{j}^{\prime}$.

On comparison of the two cases, a particularly significant difference is the absence of a weight $-1^{+}$component for linear (plane) polarization.

Returning to Eq. (3), by employing the rules of parity and angular momentum coupling, defined by $\left|j_{1}-j_{2}\right| \leqslant j \leqslant j_{1}+j_{2}$, and recognizing that the result must be a vector, which carries a weight-1 contribution only, the following is determined:

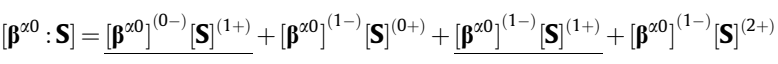

$$
\begin{aligned}
& +\underline{\left[\boldsymbol{\beta}^{\alpha 0}\right]^{(2-)}[\mathbf{S}]^{(1+)}}+\left[\boldsymbol{\beta}^{\alpha 0}\right]^{(2-)}[\mathbf{S}]^{(2+)}+\left[\boldsymbol{\beta}^{\alpha 0}\right]^{(3-)}[\mathbf{S}]^{(2+)},
\end{aligned}
$$

where the underlined terms denote contributions involving $[\mathbf{S}]^{(1+)}$, which disappear if the stimulus beam is plane polarized. For such a case, it becomes evident that the weight $-0^{-}$contributions from the material response tensor are no longer participating in the absorption mechanism.

Since one-photon allowed processes require three-photon events to also be symmetry permissible (although three-photon 
allowed systems may be one-photon forbidden as required by this Letter), normally inaccessible electronic states may become active under certain symmetries. Examples include, $B_{1}$ electronic transitions in molecules with $D_{2 d}$ symmetry, such as allene and tetrasulfur tetranitride, or $A_{1 u}$ transitions in $O_{h}$ symmetry species like sulfur hexafluoride and cubane. In molecules of the former symmetry class, the result given by Eq. (7) contains weight- $0^{-}$and weight$2^{-}$transition contributions - but no weight- $1^{-}$. Physically, this denotes that the (usually forbidden) one-photon absorption, from the totally symmetric ground state to electronic excited states, becomes possible on input of the stimulus beam. The other instance $\left(O_{h}\right.$ symmetry) delivers a weight $-0^{-}$contribution only; transitions here are therefore activated solely by input of a circularly polarized off-resonant beam. A complete list of electronic transitions and their point groups, photo-activated via the laser-modified onephoton mechanism, are shown in Table 1.

\section{Laser-modified two-photon absorption}

\subsection{Expression for efficiency}

Proceeding analogously to the previous section, the efficiency for laser-modified two-photon absorption emerges as $\Gamma_{2 \phi} \propto\left|M_{F I}^{(2)}+M_{F I}^{(4)}\right|^{2}$, where $M_{F I}^{(2)}$ and $M_{F I}^{(4)}$ are quantum amplitudes for the second- and fourth-order interaction processes, respectively. The efficiency for laser-modified two-photon absorption is expressed as [4]:

$$
\begin{aligned}
\Gamma_{2 \phi} & =\frac{2^{1 / 2} \pi \rho I}{c h \varepsilon_{0}} e_{i} e_{j} \bar{e}_{k} \bar{e}_{l}\left[\alpha_{i j}^{(0) \alpha 0} \bar{\alpha}_{k l}^{(0) \alpha 0}+\left(I^{\prime} / c \varepsilon_{0}\right) \alpha_{i j}^{(0) \alpha 0} \bar{\alpha}_{k l}^{(2) \alpha 0}\left(\omega^{\prime}\right)\right. \\
& \left.+\left(I^{\prime 2} / 4 c^{2} \varepsilon_{0}^{2}\right) \alpha_{i j}^{(2) \alpha 0}\left(\omega^{\prime}\right) \bar{\alpha}_{k l}^{(2) \alpha 0}\left(\omega^{\prime}\right)\right] .
\end{aligned}
$$

where $\boldsymbol{\alpha}^{(0) \alpha 0} \equiv \boldsymbol{\alpha}^{\alpha 0}$, in which the superscript 0 denotes a zeroth order (stimulus beam independent) contribution, and $\boldsymbol{\alpha}^{(2) \alpha 0}\left(\omega^{\prime}\right) \equiv$ $\mathbf{e}^{\prime} \overline{\mathbf{e}}^{\prime} \chi^{\alpha 0}\left(\omega^{\prime}\right)$ relates to a contribution quadratic in the electric field of the stimulus beam. The first term in Eq. (8) corresponds to two-photon absorption, independent of the stimulus beam, and the third term signifies absorption in the presence of the elastically forward-scattered stimulus beam. Again it is the second, quantum interference term (linear in $I^{\prime}$ ) that represents the leading correction.

\subsection{Population of 'two-photon forbidden' states}

A similar analysis is now undertaken for the case of two-photon absorption with Eq. (8) as the starting point. Here, the rank-2 evenparity tensors of $\boldsymbol{\alpha}^{(0) \alpha 0}$ and $\boldsymbol{\alpha}^{(2) \alpha 0}\left(\omega^{\prime}\right)$ are the important factors to consider. The latter is recast as:

$\alpha_{i j}^{(2) \alpha 0}\left(\omega^{\prime}\right) \equiv\left[\chi^{\alpha 0}\left(\omega^{\prime}\right): \mathbf{S}\right]_{i j}$,

Table 1

Electronic transitions, and their point groups, that are single-photon forbidden by symmetry, but are photo-activated on application of the stimulus beam. Columns on the right-hand side only occur when the stimulus beam is circularly polarized.

\begin{tabular}{lclc}
\hline Point group & Symmetry transition & Point group & Symmetry transition \\
\hline $0^{-}$and 2- & weight & \multicolumn{2}{c}{$0^{-}$weight only } \\
$C_{n v}$ & $A_{2}$ & $T$ & $A$ \\
$D_{n}$ & $A_{1}$ & $T_{h}$ & $A_{u}$ \\
$D_{2 h}$ & $A_{u}$ & $T_{d}$ & $A_{2}$ \\
$D_{3 h}, D_{5 h}$ & $A_{1}^{\prime \prime}$ & $O$ & $A_{1}$ \\
$D_{4 h}, D_{6 h}$ & $A_{1 u}$ & $O_{h}$ & $A_{1 u}$ \\
$D_{2 d}, D_{4 d}, D_{6 d}$ & $B_{1}$ & $I$ & $A$ \\
$D_{3 d}, D_{5 d}$ & $A_{1 u}$ & $I_{h}$ & $A_{u}$ \\
$C_{\infty v}$ & $\Sigma^{-}$ & & \\
$D_{\infty h}$ & $\Sigma_{u}^{-}$ & & \\
\hline
\end{tabular}

where $\chi^{\alpha 0}\left(\omega^{\prime}\right)$, the rank- 4 material response tensor, may be decomposed into irreducible parts of weight $0^{+}, 1^{+}, 2^{+}, 3^{+}$and $4^{+}$, so that:

$\chi^{\alpha 0}=\left[\chi^{\alpha 0}\right]^{(0+)}+\left[\chi^{\alpha 0}\right]^{(1+)}+\left[\chi^{\alpha 0}\right]^{(2+)}+\left[\chi^{\alpha 0}\right]^{(3+)}+\left[\chi^{\alpha 0}\right]^{(4+)}$,

where the final term denotes a natural fourth rank tensor (i.e. a tensor whose weight equals its rank). Again using the rules of parity, and recognizing that the result is a rank-2 tensor containing weight- 0 and weight- 2 contributions, the following is found:

$$
\begin{gathered}
{\left[\chi^{\alpha .0}: \mathbf{S}\right]=\left[\chi^{\alpha 0}\right]^{(0+)}[\mathbf{S}]^{(0+)}+\left[\chi^{\alpha 0}\right]^{(0+)}[\mathbf{S}]^{(2+)}+\underline{\left[\chi^{\alpha 0}\right]^{(1+)}[\mathbf{S}]^{(1+)}}} \\
+\left[\chi^{\alpha 0}\right]^{(1+)}[\mathbf{S}]^{(2+)}+\left[\chi^{\alpha 0}\right]^{(2+)}[\mathbf{S}]^{(0+)}+\underline{\left[\chi^{\alpha 0}\right]^{(2+)}[\mathbf{S}]^{(1+)}} \\
+\left[\chi^{\alpha 0}\right]^{(2+)}[\mathbf{S}]^{(2+)}+\underline{\left[\chi^{\alpha 0}\right]^{(3+)}[\mathbf{S}]^{(1+)}}+\left[\chi^{\alpha 0}\right]^{(3+)}[\mathbf{S}]^{(2+)} \\
+\left[\chi^{\alpha 0}\right]^{(4+)}[\mathbf{S}]^{(2+)}
\end{gathered}
$$

where the underlined terms denote contributions that disappear if the stimulus beam is plane polarized. Under the latter conditions, all weight contributions of $\chi^{\alpha 0}$ feature in the result so that, in contrast to the single-photon case, there is no experimental advantage to be gained by using a circularly polarized laser.

For certain symmetries it is again possible to achieve the optical activation of states for which conventional two-photon absorption is forbidden. To find examples of such electronic transitions, which can become allowed on input of the stimulus beam, we focus on weights $1^{+}, 3^{+}$and $4^{+}$contributions as appear in Eq. (11) (weights $0^{+}$and $2^{+}$being omitted since they already support two-photon absorption). Examples include $T_{1}$ transitions in $T_{d}$ point groups such as phosphorus pentoxide and adamantane, $A_{2}$ in $D_{4 d}$ groups like dimanganese decacarbonyl, and $E_{2}^{\prime \prime}$ in $D_{5 h}$ groups including ruthenocene. An interesting feature arises when one-photon absorption is also symmetry allowed. Physically, this represents a state previously unavailable by two-photon absorption, although permissible by a single photon equivalent, that becomes accessible on application of the stimulus beam; the ruthenocene example is one such case. A complete list of electronic transitions and their

\begin{tabular}{|c|c|c|c|}
\hline Point group & $\begin{array}{l}\text { Symmetry } \\
\text { transition }\end{array}$ & Point group & $\begin{array}{l}\text { Symmetry } \\
\text { transition }\end{array}$ \\
\hline \multicolumn{2}{|l|}{$1^{+}$weight only } & \multicolumn{2}{|c|}{$3^{+}$and $4^{+}$weight } \\
\hline$I$ & $T_{1}$ & $C_{6}$ & $B$ \\
\hline$I_{h}$ & $T_{1 g}$ & $S_{8}, D_{4 d}$ & $E_{1}$ \\
\hline \multicolumn{2}{|l|}{$3^{+}$weight only } & $C_{3 h}$ & $A^{\prime \prime}$ \\
\hline$T_{d}, O$ & $A_{2}$ & $C_{5 h}, D_{5 h}$ & $E_{2}^{\prime \prime}$ \\
\hline$O_{h}$ & $A_{2 g}$ & $C_{6 h}$ & $B_{g}$ \\
\hline$I$ & $T_{2}$ & $D_{6}, C_{6 v}$ & $B_{1}, B_{2}$ \\
\hline$I_{h}$ & $T_{2 g}$ & $D_{3 h}$ & $A_{1}^{\prime \prime}, A_{2}^{\prime \prime}$ \\
\hline \multicolumn{2}{|l|}{$4^{+}$weight only } & $D_{6 h}$ & $B_{1 g}, B_{2 g}$ \\
\hline$S_{8}$ & $B$ & $D_{6 d}$ & $E_{3}$ \\
\hline$C_{5 h}, D_{5 h}$ & $E_{1}^{\prime}$ & $C_{\infty v}$ & $\Phi$ \\
\hline$D_{4 d}$ & $B_{1}, B_{2}$ & $D_{\infty h}$ & $\Phi_{\mathrm{g}}$ \\
\hline$D_{6 d}$ & $E_{4}$ & $I$ & $G^{\circ}$ \\
\hline$C_{\infty V}$ & $\Gamma$ & $I_{h}$ & $G_{g}$ \\
\hline$D_{\infty h}$ & $\Gamma_{\mathrm{g}}$ & \multirow{2}{*}{\multicolumn{2}{|c|}{$1^{+}, 3^{+}$and $4^{+}$weight }} \\
\hline \multicolumn{2}{|c|}{$1^{+}$and $3^{+}$weight } & & \\
\hline$C_{5 v}, C_{6 v}$ & $A_{2}$ & $D_{3}, D_{4}, C_{3 v}$, & $A_{2}$ \\
\hline$D_{5}, D_{6}, D_{4 d}, D_{6 d}$ & $A_{2}$ & $D_{4 h}, D_{3 d}$ & $A_{2 g}$ \\
\hline$D_{3 h}, D_{5 h}$ & $A_{2}^{\prime}$ & $T_{d}, O$ & $T_{1}$ \\
\hline$D_{6 h}, D_{5 d}$ & $A_{2 g}$ & $O_{h}$ & $T_{1 g}$ \\
\hline$D_{4 d}, D_{6 d}$ & $A_{2}$ & & \\
\hline$C_{\infty V}$ & $\Sigma^{-}$ & & \\
\hline$D_{\infty h}$ & $\Sigma_{g}^{-}$ & & \\
\hline
\end{tabular}
point groups, activated by the laser-modified two-photon process, is given in Table 2 .

\section{Table 2}

Electronic transitions, and their point groups, that are two-photon forbidden by symmetry, but are photo-activated on application of the stimulus beam. 


\section{Discussion}

This Letter details an all-optical method, involving the input of an off-resonant stimulus beam of sufficient intensity, to promote excitation to states that are normally symmetry forbidden. The selection rules procedure we have employed follows a pattern established previously on nonlinear optical processes, for example Refs. [12-18], and considers the irreducible Cartesian tensor components of the polarization and molecular response tensors [11]. An irreducible Cartesian basis affords direct connectivity with the directional properties of the radiation and of the electronic transitions, while also affording a transparent linkage with the symmetry-based irreducible representations of the molecular electronic states, using the rules of angular momentum coupling.

In general, it is anticipated that experimental observations of laser-modified absorption will most readily discernible in molecules and complexes belonging to one of the cubic point groups - those of high symmetry number, with interchangeable Cartesian axes but not necessarily a center of symmetry (a property of the tetrahedral groups, for example). Since this type of high symmetry allows for isotropic polarizability, the molecules will not be oriented by the strong fields of the stimulus radiation, which might otherwise produce complications through photoselection effects. Molecules of such symmetry offer the greatest scope for exploiting states and transitions of varying symmetry. Moreover, cubic symmetry species mostly have electronic excited states similar in structure to the ground state - except for cases where Jahn-Teller effects are prominent.

Lastly we note that, although the passage of the off-resonant beam through the sample might, in principle, expedite direct fluorescence decay in a way that once again circumvents the usual selection rules (the theory has been developed in a number of recent studies [19-21]), it is the case that many longer lived or metastable excited states will remain populated beyond the throughput of the off-resonant beam, enabling the system to be sustained in a state of electronic excitation that is normally unachievable. We consider that this offers interesting prospects for potential energy trapping and other novel kinds of photochemistry.

\section{Acknowledgements}

The authors are grateful to the Leverhulme Trust for funding this research.

\section{References}

[1] D.P. Craig, T. Thirunamachandran, Molecular Quantum Electrodynamics: An Introduction to Radiation-Molecule Interactions, Dover Publications, Mineola, NY, 1998.

[2] A. Salam, Molecular Quantum Electrodynamics: Long-Range Intermolecular Interactions, Wiley, Hoboken, NJ, 2009.

[3] L. Mandel, E. Wolf, Optical Coherence and Quantum Optics, Cambridge University Press, Cambridge, NY, 1995.

[4] D.S. Bradshaw, D.L. Andrews, Phys. Rev. A 88 (2013) 033807.

[5] J.A.R. Coope, R.F. Snider, F.R. McCourt, J. Chem. Phys. 43 (1965) 2269.

[6] J.A.R. Coope, R.F. Snider, J. Math. Phys. 11 (1970) 1003.

[7] J. Jerphagnon, Phys. Rev. B 2 (1970) 1091.

[8] A.J. Stone, Mol. Phys. 29 (1975) 1461.

[9] A.J. Stone, J. Phys. B: At. Mol. Opt. Phys. 9 (1976) 485.

[10] J. Jerphagnon, D. Chemla, R. Bonneville, Adv. Phys. 27 (1978) 609.

[11] D.L. Andrews, P. Allcock, Optical Harmonics in Molecular Systems, Wiley-VCH, Weinheim, 2002.

[12] D.L. Andrews, N.P. Blake, J. Phys. A: Math. Gen. 22 (1989) 49.

[13] S. Brasselet, J. Zyss, J. Opt. B: Quantum Semiclass. Opt. 15 (1998) 257.

[14] C. Andraud, T. Zabulon, A. Collet, J. Zyss, Chem. Phys. 245 (1999) 243.

[15] R. Piron, S. Brasselet, D. Josse, J. Zyss, G. Viscardi, C. Barolo, J. Opt. B: Quantum Semiclass. Opt. 22 (2005) 1276.

[16] D.L. Andrews, Phys. Rev. A 81 (2010) 033825.

[17] S.N.A. Smith, D.L. Andrews, J. Phys. A: Math. Gen. 44 (2011) 395001.

[18] T. Bancewicz, J. Math. Chem. 50 (2012) 1570.

[19] D.S. Bradshaw, D.L. Andrews, J. Phys. Chem. A 113 (2009) 6537.

[20] D.S. Bradshaw, D.L. Andrews, Phys. Rev. A 81 (2010) 013424.

[21] J.M. Leeder, D.S. Bradshaw, D.L. Andrews, J. Phys. Chem. B 115 (2011) 5227. 